\title{
Efectividad de la administración oral versus intramuscular de vitamina B12 en pacientes con deficiencia: revisión sistemática
}

\author{
Efectiviness of the oral administration \\ versus intramuscular of B12 vitamin in patients \\ with deficiency: systematic review
}

\begin{abstract}
Vitamin B12 or cobalamin deficiency is a very common condition and is one of the most important causes of anemia. It can be treated with oral or intramuscular treatment. A summary of the evidence will be made through a systematic review of randomized clinical trials that have compared both treatments for the management of these patients. The objective of this review was to determine whether there is scientific evidence supporting the effectiveness of oral versus intramuscular vitamin B12 treatment of its deficiency. The search strategy included randomized clinical trials (RCTS); databases used were: Medline, Cochrane Central, Lilacs and CINAHL. Also an electronic search in Google Scholar was performed. The results were three articles that met our eligibility criteria. We conclude that there is moderate evidence that the administration of oral vitamin B12 is most effective in the medium term increment serum cobalamin volume in patients with vitamin B12 deficiency.

Key words: vitamin B12 deficiency, anemia, oral cobalamin treatment, intramuscular cobalamin treatment.
\end{abstract}

Felipe Araya Q.

Helga Arias $S$.

Miguel Hernández

Úrsula Inostroza

María José Urzúa

Escuela de Nutrición y Dietética. Facultad de Ciencias de la Salud Universidad de las Américas. Santiago, Chile

Dirigir la correspondencia a: Felipe Araya Quintanilla. Universidad de las Américas. Avda. Manuel Montt 948. Santiago de Chile Chile. fandres.kine@gmail.com

Este trabajo fue recibido el 15 de Diciembre de 2015 y aceptado para ser publicado el 20 de Abril de 2016.

\section{INTRODUCCIÓN}

La deficiencia de la vitamina B12 es una condición muy común (1). En la población general, la prevalencia se estima entre 1,5 a $15 \%$ (2). Esta deficiencia puede causar principalmente anemia y también otras enfermedades neuropsiquiátricas como es el caso de las parestesias y ataxias (3). La anemia, es una de las consecuencias más importantes del déficit de cobalamina, se puede producir por tres mecanismos patogénicos independientes, en ocasiones combinados entre sí: por disminución de la producción medular de glóbulos rojos, asociado al déficit de B12, por aumento de la destrucción de glóbulos rojos y por pérdidas hemáticas (4). La deficiencia de la vitamina B12 se da principalmente por una malabsorción de esta vitamina adquirida en los alimentos, la que incluye 50\% de los casos, especialmente en los ancianos por hipo o aclorhidia, ya sea farmacológica o por patología gástrica, y la anemia perniciosa, por déficit de factor intrínseco, proteína que se produce en el revestimiento del estómago (5). La vitamina B12 sólo se encuentra de manera natural en alimentos de origen animal.
Los animales incorporan la B12 en sus tejidos, huevos y leche. Artificialmente se encuentra en alimentos fortificados y suplementos (6) .Por todos estos factores, la anemia puede ser considerada como una de las patologías típicas en el paciente geriátrico. Es una enfermedad potencialmente reversible, por lo que, en la gran mayoría de los casos, se justifica la realización de las pruebas diagnósticas para afiliar su etiología y tratarse de forma precisa (7). La anemia es entonces, el desorden hematológico más frecuente de la población. En Chile, Olivares et al informaron que, en mayores de 60 años, 50,5\% de los hombres y 33,1\% de las mujeres presentan déficit de vitamina B12 (8). Estas cifras tan dispares dependen de varios factores, como el método diagnóstico, los niveles de hemoglobina o hematocrito, el punto de corte empleado, la edad, el sexo que es mayor en varones $y$, sobre todo, el tipo de población estudiada (9).

La falta de vitamina B12 puede deberse a factores alimentarios, como consumir una dieta vegetariana; alimentación deficiente en los lactantes; desnutrición durante el 
embarazo; problemas de salud que dificulten al cuerpo la absorción de suficiente vitamina B12, como el alcoholismo crónico, la enfermedad de Crohn, la enfermedad celíaca, infección con la tenia de los peces u otros problemas que no permitan al organismo la digestión de los alimentos; cirugías para extirpar ciertas partes del estómago o el intestino delgado, como algunas cirugías para bajar de peso; uso de antiácidos y otros medicamentos para la acidez gástrica por un tiempo prolongado (10). Es posible que no se presenten síntomas o pueden ser leves, entre ellos la diarrea o estreñimiento, fatiga, falta de energía o mareo al pararse o hacer esfuerzo, inapetencia, piel pálida, problemas de concentración, dificultad respiratoria, sobre todo durante el ejercicio, inflamación y enrojecimiento y sangramiento de la lengua o encías. Si se tiene niveles bajos de vitamina B12 por mucho tiempo, se pueden presentar daños neurológicos. Los síntomas abarcan desde confusión o cambio en el estado mental (demencia) en casos graves, depresión, pérdida del equilibrio, entumecimiento y hormigueo de manos y pies (11).

El proceso de absorción de B12 puede verse afectado a nivel del estómago, donde se produce el factor intrínseco que es el que permite la absorción de la cobalamina. La deficiencia causa anemia, fatiga, disturbios en el humor y otras complicaciones neuropsiquiátricas y neurológicas. Y también ha sido asociada a un incremento de riesgo de infarto y ataque al corazón (12).

Tradicionalmente, la sustitución de la vitamina B12 deficitaria ha sido administrada por vía intramuscular (IM) porque la absorción del tracto gastrointestinal es deficiente. La mayoría de los individuos con deficiencia de vitamina B12 en el Reino Unido y en otros países son tratados con vitamina B12 intramuscular. La vitamina B12 intramuscular se puede administrar de dos formas diferentes: cianocobalamina e hidroxocobalamina. En algunos países, la hidroxocobalamina ha reemplazado completamente a la cianocobalamina como primera elección para el tratamiento con vitamina B12, porque se retiene más tiempo en el cuerpo y se puede administrar a intervalos de hasta 3 meses (13). Las inyecciones intramusculares son una fuente considerable de trabajo para los profesionales de asistencia sanitaria, principalmente los médicos generales y las enfermeras de la comunidad (14), y pueden ocasionar dolores significativos en pacientes delgados (15). Aunque las reacciones adversas son raras, las inyecciones pueden ser peligrosas en pacientes anticoagulados. Hay poca diferencia en el costo del tratamiento oral versus intramuscular cuando se considera únicamente la medicación. Sin embargo, la administración intramuscular a menudo incluye un viaje especial a un establecimiento de salud o una visita domiciliaria por un profesional de la salud para aplicar la inyección (16). Por consiguiente, el tratamiento (tto) oral permitiría ahorrar considerables recursos del Servicio de Salud. El tratamiento puede incluir una inyección de vitamina B12 una vez al mes. Las personas con niveles muy bajos de vitamina B12 pueden necesitar al principio más inyecciones al principio. Algunos pacientes también deben tomar suplementos de vitamina B12 por vía oral. Para ciertas personas, las tabletas de altas dosis de vitamina B12 tomadas por vía oral funcionan bien y las inyecciones no son necesarias (17).

La vitamina B12 es un cofactor para sólo 2 enzimas: la metionina sintetasa y la L-metilmalonil-coenzima A mutasa. La anemia megaloblástica que se observa en ambas deficiencias, se debe a la interacción entre el folato y la vitamina B12. La alteración de la sincronía entre la maduración del citoplasma y el núcleo conduce a la macrocitosis, núcleos inmaduros e hipersegmentación de los granulocitos en la sangre periférica (18). La vitamina B12 tiene un papel esencial precisamente en la síntesis de ADN. Cuando existe una carencia seria se deteriora la capacidad de síntesis de ADN de las células, capacidad que en principio es esencial para que exista multiplicación celular. Así, la carencia de vitamina B12 conlleva una ralentización de la producción de las células tanto más acusada cuánto más proliferación celular exista en el tejido del que se trate.

La anemia perniciosa requiere una reposición de vitamina B12 de por vida, casi siempre usando inyecciones. Algunos pacientes pueden obtener suficientes suplementos con tabletas de vitamina B12 oral en dosis altas, en cuanto la anemia causada por absorción y digestión deficiente se trata con inyecciones de vitamina B12 hasta que el trastorno mejore. Estas inyecciones se aplican todos los días, luego cada semana al principio y luego cada mes (19).

La anemia es una entidad nosológica de gran importancia en las personas mayores. Su prevalencia es alta, y su incidencia se incrementa con la edad, especialmente en mayores de 85 años, segmento de la población que es el que proporcionalmente estará creciendo más en las próximas décadas. Puede inferir en el funcionamiento de varios órganos y sistemas, relacionándose con un aumento de la morbilidad, la mortalidad y del déficit funcional (20-22).

Por otro lado, los vegetarianos estrictos y los lactantes nacidos de madres deficientes en B12 constituyen grupos de alto riesgo (23). El estado nutricional de B12 puede ser pobre al evitar alimentos de origen animal específicos como en el lacto ovo vegetarianismo o al ingerir pocos alimentos de origen animal por razones culturales o económicas (24).

El objetivo de este trabajo fue poder determinar mediante evidencia científica, qué terapia es más efectiva en la administración de vitamina B12, de manera oral versus intramuscular.

\section{OBJETIVO}

Determinar si existe evidencia científica que avale la efectividad de la administración oral versus intramuscular de la vitamina B12 para el tratamiento de su deficiencia.

\section{METODOLOGÍA \\ Estrategias de búsqueda}

La búsqueda se realizó en septiembre de 2015 y se utilizaron algunos términos $\mathrm{MeSH}$, Tesauro de PubMed como: vitamine B12, anemia, cobalamin.

Para llevar a cabo la búsqueda en la base de datos MEDLINE se utilizó la estrategia de búsqueda sensible propuesta en el "Handbook Cochrane" (25).

\section{Bases de datos}

La búsqueda se realizó el día 12 de setiembre de 2015, donde fueron incluidas las bases de datos Medline (http:// www.ncbi.nlm.nih.qov/pubmed), Cochrane Central (http:// www.cochrane.org/), Lilacs (www.bases.bireme), CINAHL (https://health.ebsco.com/products/the-cinahl-database), y también se revisaron otras fuentes de búsqueda como Google Scholar (https://scholar.google.cl/). Los detalles de la selección de artículos se encuentran en la figura 1.

\section{Términos de búsqueda}

Los términos de búsqueda de nuestra revisión fueron obtenidos de los MeSH, a través de tesauro de PubMed, siendo algunos de ellos, anemia, cobalamin, entre otros.

1. Vitamin B12 deficiency 
2. Anemia

3. Pernicious

4. Vitamin B12

5. (\#1) OR (\#2) OR (\#3) OR (\#4)

6. Cobalamin supplement vitamin B12

7. Oral administration

8. Intramuscular administration

9. (\#6) OR (\#7) OR (\#8)

10. (\#5) AND (\#9)

11. Randomized clinical trial

12. Clinical trial

13. (\#11) OR (\#12)

14. Humans

15. Animals

16. (\#14) NOT (\#15)

17. (\#10) AND (\#13) AND (\#16)

Límites de búsqueda

- Ensayos clínicos aleatorizados y controlados

- $\quad$ Sin distinción de género y raza

- Artículos publicados en idioma inglés o español

- Artículos publicados desde el 01 de enero 1980 hasta el 10 de septiembre de 2015

Criterios de selección

A los artículos seleccionados por la búsqueda preliminar, se les aplicó una revisión crítica y éstos fueron evaluados según los siguientes criterios:
Criterios de inclusión

- Estudios que hayan investigado la suplementación oral de la vitamina B12

- Estudios con pacientes con diagnóstico de anemia o deficiencia de B12

- Estudios que hayan investigado la administración IM de la vitamina B12

- $\quad$ Estudios que evalúen o midan el volumen sérico de B12.

Criterios de exclusión

- Estudios en fase preliminar o protocolo.

- Estudios con diseño crossover.

Evaluación de la calidad metodológica

Se realizó la evaluación de la calidad metodológica según la escala de PEDro (26).

Un estudio se considera de baja calidad metodológica si obtiene un puntaje de 4 o menos puntos (27). Si obtiene 5 puntos, tiene una moderada calidad metodológica. Y, si obtiene de 6 hasta 10 puntos $(28,29)$, se considera de alta calidad metodológica. La evaluación de la misma se encuentra en la tabla 1.

Recopilación de datos

Dos de los autores (HA y UI) realizaron la búsqueda de todos los artículos en forma independiente, quienes además evaluaron la calidad metodológica de los artículos seleccio-

\section{FIGURA 1}

Algoritmo de búsqueda

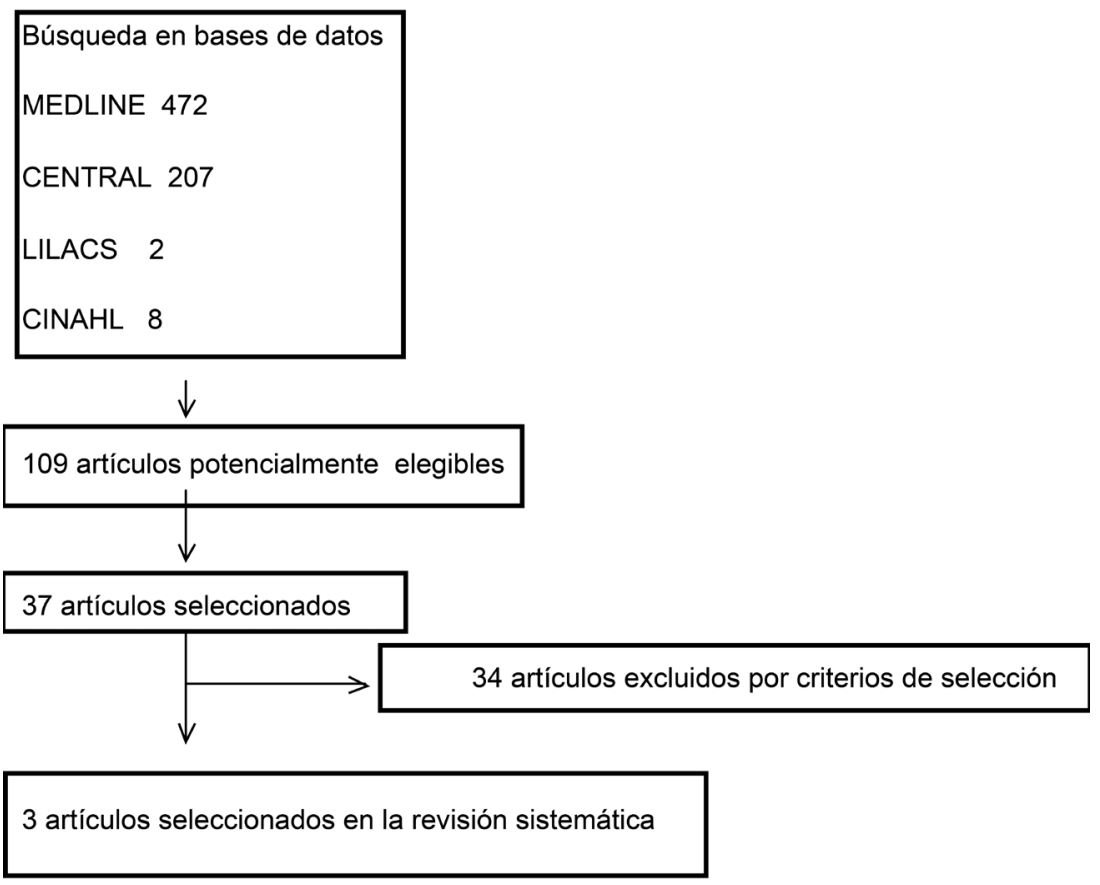


nados con la escala de PEDro. Se llamó a un tercer asesor independiente (FA) para discernir acerca de los puntos que estaban en conflicto, para decidir mediante discusión y consenso su inclusión final y el análisis de los puntos relevantes.

Síntesis y análisis de datos

De acuerdo a los análisis de datos de los estudios que cumplieron los criterios de elegibilidad se realizó la siguiente comparación: vitamina B12 intramuscular en comparación con vitamina B12 en forma oral. Para ello, se analizaron 3 artículos (30-32). Para el análisis de datos se utilizó el programa Rev-Man 5. Las medidas de resultados funcionales fueron analizadas como variables contínuas; los estimadores puntuales ocupados para variables contínuas como diferencia de medias estandarizadas (DME), con sus respectivos intervalos de confianza 95\% (IC). Los resultados de los artículos sólo se pueden combinar para llegar a un estimador puntual cuando son homogéneos y estos fueron Bolaman (31), Castelli (33) y Kuzminski (30). Además, se evaluó la homogeneidad clínica y estadística. Esta última fue evaluada con la prueba estadística de chi $^{2}$ y el test de heterogeneidad $\mathrm{I}^{2}$. Se consideró admisible realizar un metaanálisis, cuando el análisis de los datos mostraban baja heterogeneidad, con un valor $X^{2}$ con un $P>0,1$ y el test $I^{2}$ con un valor menor o igual a 40\%. Los resultados del metaanálisis son -0.33 IC $(-0.66,0.01)$, para la comparación de vitamina B12 oral e IM en volumen sérico al cuarto mes.

Criterios para valoración de los resultados

Para el resto de las comparaciones realizadas por nuestra revisión, no existía homogeneidad clínica que permitiera agrupar los datos de algunos de los estudios según un estimador puntual, por lo tanto, se realizó un metaanálisis. No obstante,

\section{TABLA 1}

Valoración de los artículos incluidos en base a la escala de PEDro.

\begin{tabular}{|c|c|c|c|c|c|c|c|c|c|c|c|c|}
\hline Autor & $\mathbf{2}$ & $\mathbf{2}$ & $\mathbf{3}$ & $\mathbf{4}$ & $\mathbf{5}$ & $\mathbf{6}$ & $\mathbf{7}$ & $\mathbf{8}$ & $\mathbf{9}$ & $\mathbf{1 0}$ & $\mathbf{1 1}$ & Total \\
\hline $\begin{array}{c}\text { Castelli } \\
2011\end{array}$ & sí & sí & sí & sí & no & no & no & sí & sí & sí & sí & $7 / 10$ \\
\hline $\begin{array}{c}\text { Bolaman } \\
2013\end{array}$ & sí & sí & no & sí & no & no & no & sí & sí & sí & sí & $6 / 10$ \\
\hline $\begin{array}{c}\text { Kuzminski } \\
1998\end{array}$ & sí & sí & no & sí & no & no & no & sí & sí & sí & sí & $6 / 10$ \\
\hline
\end{tabular}

\section{FIGURA 2}

Forest plot B12 oral versus IM volumen sérico B12 al cuarto mes.

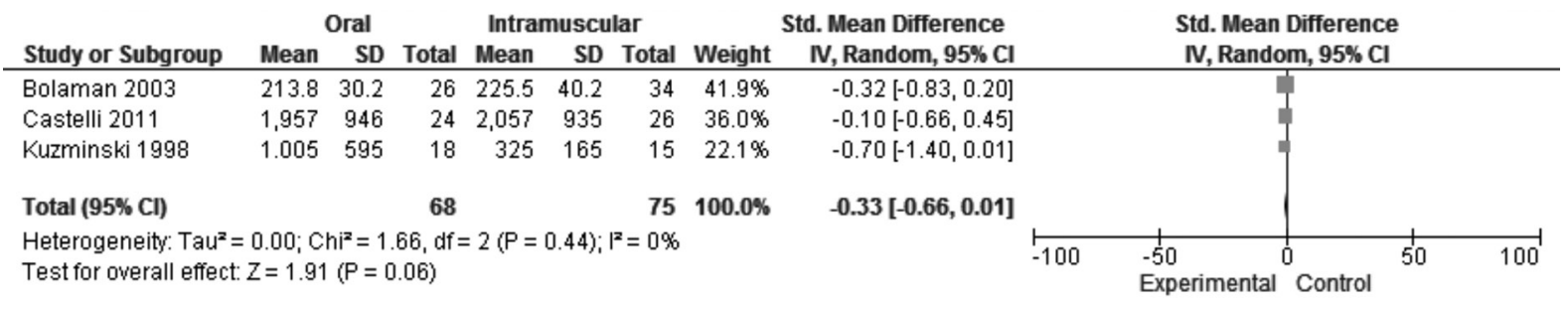


la conclusión será en base a un método cualitativo recomendado por el Grupo Cochrane de Espalda (Van Tulder, 2003) (34) con el uso de niveles de evidencia para la síntesis de los datos.

- $\quad$ Evidencia sólida: proporcionada por hallazgos generalmente consistentes en múltiples ECAs (3 o más) de alta calidad metodológica.

- Evidencia moderada: proporcionada por resultados generalmente consistentes en 1 ECA de alta calidad metodológica, también se puede interpretar como hallazgos generalmente consistentes en múltiples ECAs con moderada calidad metodológica.

- $\quad$ Evidencia limitada: proporcionada por hallazgos consis- tentes en 1 o más ECAs de baja calidad metodológica.

- Evidencia contradictoria: hallazgos no coherentes o inconsistentes en múltiples ECAs.

- Ninguna evidencia: ningún ECA encontrado.

\section{RESULTADOS}

Selección y características de los estudios

De acuerdo con nuestros criterios de revisión, la búsqueda preliminar identificó 689 artículos potencialmente elegibles. Al aplicar los límites de búsqueda y los criterios de selección, quedaron 37 estudios en un primer lugar, para finalmente

\section{TABLA 2}

Características de artículos incluidos.

\begin{tabular}{|c|c|c|c|c|}
\hline Autor/año & Condición/método & Características de los pacientes & Intervención & Seguimiento/resultados \\
\hline $\begin{array}{l}\text { Castelli } M^{\circ} \\
\text { Cristina (33) } \\
(2011)\end{array}$ & $\begin{array}{l}\text { Estudio Randomi- } \\
\text { zado, con sobre } \\
\text { abierto, en grupos } \\
\text { paralelos y generados } \\
\text { en computadores } \\
\text { usando randomiza- } \\
\text { ción en bloques. }\end{array}$ & $\begin{array}{l}\mathrm{n}=50 \text { personas } \\
\text { (11 hombres y } 39 \\
\text { mujeres) } \\
\text { Grupo } 1 \text { Oral B12 } \mathrm{n}=24 \\
\text { Grupo 2, Intramuscular } \\
\text { B12, } \mathrm{n}=26\end{array}$ & $\begin{array}{l}\text { Durante } 3 \text { meses a pacien- } \\
\text { tes entre } 18-60 \text { años. } \\
\text { Grupo 1: } 1 \text { tableta oral en } \\
\text { ayuna de } 1000 \text { ug con ml } \\
\text { de agua. } \\
\text { Grupo 2: } \\
9 \text { inyecciones intramuscu- } \\
\text { lares }\end{array}$ & $\begin{array}{l}\text { Para ambas terapias } \\
\text { es efectivo el tto para } \\
\text { normalizar los niveles } \\
\text { séricos de cobalamina } \\
\text { en todos los pacientes. } \\
\text { Ambos ttos fueron bien } \\
\text { tolerados en las dosis } \\
\text { estudiadas. }\end{array}$ \\
\hline
\end{tabular}

De 1000 ug por 3 meses.

96\% de los pacientes completó el estudio $\mathrm{n}=22$ para tto oral y $n=26$ para tto intramuscular.

Bolaman Zahit Randomizado y pros(27)

(2003) pectivo con sobre abierto, en computadores, usando métodos randomizados de bloques. $\mathrm{n}=60$

Grupo $1 \mathrm{n}=26$

Grupo 2 n=34

Duración=90 días. Grupo 1 Administración Oral Grupo 2, administración intramuscular.

Grupo 1: A pacientes $>0=$ 16 años recibieron 1000 ug de cobalamina oral diariamente por 10 días

Grupo 2: 1000 ug de cobalamina intramuscular $1 \mathrm{vez}$ al día por 10 días.

Después de los 10 días se les administró una vez a la semana por 4 semanas y después una vez por mes.

Intervención: 4 meses. Grupo 1: Administración oral de 2 mg de B12 a diario por 120 días.

Grupo 2: Administración intramuscular de $1 \mathrm{mg}$ de B12 en los días 1, 3, 7, 10, $14,21,30,60$ y 90 .
Resultado: una moderada dosis de vitamina B12 oral es tan efectiva como la administración intramuscular y el tto oral fue mejor tolerado y menos caro en comparación con la intramuscular.

Grupo 2 (intramuscular): $\mathrm{n}=15$, edad 71 años

Seguimiento por 16 semanas.

Como resultado, los dos grupos no difirieron significativamente en el aumento de los niveles de B12 después de los tratamientos, ya sean de forma oral o de forma intramuscular. 
seleccionar tres de ellos. De los tres estudios seleccionados el número de pacientes fue 148 . Todos se realizaron en pacientes con anemia. El promedio de pacientes por estudio fue 49,3, el rango de edad de los pacientes estaba entre los 47,5 y 71 años con un promedio de 59,3 años. El resumen de las características clínicas y metodológicas de los artículos incluidos se encuentra en la tabla 2.

Medidas de resultados

Concentración sérica de vitamina B12 medido con perfil sanguíneo $(\mathrm{pg} / \mathrm{mL})$.

\section{Intervenciones y comparaciones}

Las intervenciones terapéuticas estudiadas fueron la aplicación de la vitamina B12 de manera oral en comparación con la intramuscular. Para ello, se analizó la efectividad de estos tratamientos en tres artículos que correspondían con nuestros criterios de estudio (31-33-30).

\section{Resultados de intervenciones}

Tres estudios (31-33-30) con un $n=148$ pacientes, investigaron la efectividad de la administración de la vitamina B12 de forma oral en comparación con la forma intramuscular. El resumen de todos los aspectos metodológicos de los artículos y características clínicas se encuentran en la tabla 2.

\section{Administración de vitamina B12 oral en comparación con intramuscular}

En el estudio de Bolaman et al del año 2013 (31), se investigó la eficacia del tratamiento oral e intramuscular de vitamina B12 en pacientes mayores de 16 años con diagnóstico de anemia megaloblástica con déficit de cobalamina, durante 90 días. Los pacientes de ambos grupos, oral e intramuscular con déficit de cobalamina recibieron 1000 ug una vez al día durante 10 días; luego recibieron el tratamiento una vez a la semana por 4 semanas, y finalmente una vez al mes de por vida. El estudio demostró que ambos tratamientos son igual de efectivos. Sin embargo, el tratamiento oral resultó ser más económico y mejor tolerado por los pacientes, a pesar que con respecto a los resultados, el grupo intramuscular obtuvo mejores puntuaciones. Al día 90 de tratamiento los valores de vitamina sérica en el grupo oral fueron en promedio de 213.8 (30.2) y en el grupo intramuscular 225.5 (40.2).

El estudio de Castelli et al del año 201133, tuvo por objetivo comparar la eficacia del tratamiento oral e intramuscular de vitamina B12 en base a concentraciones séricas < 350 pg/ $\mathrm{mL}$. Ambos grupos recibieron una dosis de B12 de $1000 \mathrm{ug}$ durante 3 meses. El grupo oral, lo tomó diariamente por 90 días y el grupo intramuscular en los días 1, 3, 7, 10, 14, 21, 30, 60 y 90. Los pacientes tenian entre 18 y 60 años, y más. Ambos grupos fueron efectivos con respecto a la normalización de los niveles séricos de vitamina B12 y a la tolerancia por los pacientes. Pese a esto, el grupo de administración intramuscular obtuvo mayores niveles en promedio de concentración de volumen sérico, de 2057 (935), y el grupo oral de 1957 (946).

El estudio de Kuzminski et al del año 1998 30, investigó la eficacia del tratamiento oral en 38 pacientes randomizados diagnosticados con deficiencia de cobalamina versus el tratamiento intramuscular. Tuvo una duración de 120 días. En el tratamiento intramuscular se administró $1 \mathrm{mg}$ de B12 los días $1,3,7,10,14,21,30,60$ y 90 y el tratamiento oral se administró $2 \mathrm{mg}$ diarios por 4 meses .Como resultado de la investigación, para la administración de manera oral, antes de la intervención se obtuvo $93+46$ pg/Ml de B12, y después del tratamiento $1,005+595 \mathrm{pg} / \mathrm{mL}$ de B12. Con respecto al tratamiento intramuscular antes de la intervención, se obtuvo $95+92$ pg7ML de cobalamina y después del tratamiento, $325+165$ pg/Ml de B12. El volumen de cobalamina sérica a los 4 meses postratamiento, demostró diferencias significativas en el grupo oral en comparación con el intramuscular $\mathrm{P}<0.005$.

\section{DISCUSIÓN}

Esta investigación intentó determinar la efectividad del tratamiento oral y el intramuscular en pacientes con deficiencia de vitamina B12. Se realizó una síntesis de la evidencia disponible para responder la interrogante planteada anteriormente. Se seleccionaron tres artículos por nuestra búsqueda, los cuales estaban en condiciones de resolver la pregunta planteada. Se realizó un metaanálisis gracias a la homogeneidad clínica y estadística.

Con respecto a la calidad metodológica de los tres estudios seleccionados, el estudio de Castelli (33) obtuvo una puntuación de 7/10 puntos de PEDro, los estudios de Bolaman (31) y Kuzminski (30) obtuvieron 6/10 puntos de PEDro, por lo tanto, los tres estudios presentan una alta calidad metodológica. Cabe señalar, que el estudio de Castelli (33) fue randomizado mediante una secuencia generada por computadores, con sobres abiertos. En el estudio de Bolaman (31) también hubo randomización en bloques y en el estudio de Kuzminski30 la randomización se hizo por análisis y sistemas por computadores. Este punto metodológico es relevante, ya que es el que entrega mayor validez a los ensayos clínicos. En los tres estudios no hubo triple ciego, ya que ni los sujetos, terapeutas ni evaluadores fueron ciegos. Los tres estudios seleccionados contemplaron la exclusión de los pacientes o el manejo adecuado por análisis de intención de tratar. Ninguno de los estudios tiene inscrito el protocolo de investigación en algún sistema de registro existente en la actualidad. Según esto, se hace muy difícil poder establecer si hubo un reporte selectivo de medidas de resultados.

Es importante destacar con respecto a la investigación existente anteriormente publicada, una revisión del tema Butler et all, el año 2006 (35). Esta revisión contempló la inclusión solamente de dos ensayos clínicos, pero no se evaluó la calidad metodológica de los estudios y la búsqueda fue deficiente y desactualizada. Existen limitantes con respecto a la síntesis de la evidencia disponible para responder la interrogante planteada anteriormente.

En cuanto a la efectividad del tratamiento intramuscular u oral, el tratamiento oral es más efectivo a mediano plazo en el volumen sérico de cobalamina en pacientes con déficit de vitamina B12. Sin embargo, ambos tratamientos muestran diferencias significativas con respecto al volumen sérico de vitamina B12 y que ambos tratamientos tuvieron efectos adversos en $>5 \%$ de pacientes según el estudio de Castelli (33). Estos efectos fueron dolor abdominal, constipación, diarrea, náuseas, fatiga, entre otros. Con respecto a las ventajas y desventajas de la terapia oral, parece evidente que la terapia puede ser bioquímicamente equivalente a la intramuscular, pero hay otros aspectos que se deben considerar. Se ha indicado que la conveniencia de los pacientes debe ser un tema a la hora de valorar cualquier cambio de terapéutica, especialmente cuando se trata de una deficiencia que afecta con frecuencia a ancianos. Se ha planteado una buena aceptación de la cobalamina oral. Otro aspecto práctico es que las inyecciones intramusculares suelen ser dolorosas, molestas y poco toleradas por muchos pacientes, sobre todo los más delgados (36). La terapia oral tendría otras ventajas para los pacientes, pues evita 
las visitas reiteradas al médico o al centro de salud para recibir las inyeciones, con los que se ahorra tiempo y dinero tanto en viajes como en menor costo del medicamento (37). Las inyecciones intramusculares además de ser dolorosas implican un riesgo para el personal de salud que las administra, ya que se pueden lesionar con las agujas usadas, además de ocupar un tiempo que podría derivarse a otros servicios médicos. Se ha señalado que el costo de la terapia parenteral para un año es considerable y el ahorro potencial por el cambio de todos los pacientes a terapia con cobalamina oral es sustancial. Este ahorro fue estimado para 5 años en cifras del orden de \$2,9 a $\$ 17,6$ millones, solamente en notario. Ahora bien, mientras que el tratamiento oral con vitamina B12 puede utilizarse por muchos pacientes, este proceder podría ser inadecuado para aquellos con alguna dificultad para el manejo de los medicamentos orales, como es el caso de los pacientes que tienen una pérdida significativa de la memoria o disfunción cognitiva (38). La terapia oral también podría ser inadecuada para los que tengan dificultad para tragar, aunque sería algo relativo, pues se ha demostrado que la administración por vía subligual sería igualmente efectiva. Otros estudios han demostrado que el régimen de dosis orales de vitamina B12 es seguro desde el punto de vista de costo y efectividad, bien tolerado y aceptado por la mayoría de los pacientes. Se ha reportado que la mayoría de los pacientes que cambian por el régimen oral se muestran satisfechos y desean continuar con él, por lo que se ha sugerido que la terapia con vitamina B12 oral debe ser considerada por los médicos como una alternativa ventajosa para las personas con deficiencias. Siempre hay que considerar el contexto en el cual se encuentra nuestro paciente para poder tomar la mejor decisión con respecto a la intervención.

\section{CONCLUSIONES}

Según los resultados arrojados por los estudios incluidos en esta revisión sistemática, existe moderada evidencia que la administración de la vitamina B12 oral es más efectiva a mediano plazo en el volumen sérico de cobalamina en pacientes con déficit de vitamina B12, cuyo objetivo es obtener respuestas hematológicas y neurológicas a corto plazo. Las investigaciones encaminadas a evaluar esta alternativa oral y el por qué aún su uso no ha sido del todo extendido, sugiere que el cambio de los pacientes al tratamiento oral con vitamina B12 implica ahorros sustanciales en términos de costos financieros, tiempo aportado por médicos y personal de la salud, siendo estos puntos los que marcan la preferencia oral. Cabe destacar que quizás haya quedado algún estudio fuera de nuestras revisiones sistemáticas en el aspecto de búsqueda.

Es importante tomar en cuenta los posibles efectos adversos que puede provocar el tratamiento y las posibles interacciones que puedan interferir en la absorción de la vitamina B12 en los pacientes. Así mismo, poder tomar las medidas necesarias y lograr una correcta decisión clínica para el tratamiento de los pacientes con déficit de vitamina B12.

\section{RESUMEN}

La deficiencia de la vitamina B12 o cobalamina es una condición muy común y es una de las causas más importantes de la anemia. Puede ser tratada con un tratamiento oral o intramuscular. Se realizó una síntesis de la evidencia a través de una revisión sistemática de ensayos clínicos aleatorizados que hayan comparado ambos tratamientos para el manejo de estos pacientes. La estrategia de búsqueda incluyó ensayos clínicos aleatorizados (ECA); las bases de datos usadas fueron: Medline, Cochrane Central, Lilacs y Cinahl. Además se realizó una búsqueda electrónica en Google Scholar. Los resultados obtenidos fueron de tres artículos que cumplían con nuestros criterios de elegibilidad. El objetivo de esta revisión fue determinar si existe evidencia científica que avale la efectividad de la administración oral versus intramuscular de la vitamina B12 para el tratamiento de su deficiencia. Se concluyó que existe moderada evidencia que la administración de la vitamina B12 oral es más efectiva que la administración intramuscular a mediano plazo en el volumen sérico de cobalamina en pacientes con déficit de vitamina B12.

Palabras clave: deficiencia de vitamina B12, anemia, tratamiento oral e intramuscular.

\section{Conflicto de interés: No declarados.}

Agradecimineos: Se agradece a los autores de la revisión por prestar información al desarrollo de esta investigación.

\section{BIBLIOGRAFÍA}

1. Van Walraven C, Naylor C. Use of vitamin $B 12$ injections among elderly patients by primary care practitioners in Ontario. Can Med Assoc J. 1999; 161: 146-9.

2. Pennypacker LC, Allen RH, Kelly JP, Matthews LM, Grigsby $J$, kaye K. High prevalence of cobalamin deficiency in elderly outpatients. J Am Geriatr Soc. 1992;40:1197-204.

3. Oh R, Brown DL. Vitamin B12 deficiency. Am Fam Physi. 2003 1;67(5):979-86.

4. Argente H. Semiología médica: Fisiopatología, Semiotecnia y Propedéutica: Enseñanza basada en el paciente/Horacio A. Argente y Marcelo E. Alvarez-1ํ ed-Buenos Aires: Médica Panamericana, 2008.

5. Dharmajaran TS, Audiga GU, Norkus EP. Vitamin B12 deficiency. Recognizing subtle symptoms in older adults. Geriatrics 2003;58:30-8.

6. Allen LH. Causes of vitamin B12 and folate deficiency. Food Nutr Bull. 2008;29(2):20-34.

7. Carmel R. Anemia and aging: an overview of clinical diagnostic and biological issues. Blood Rev. 2001; 15.9-18.

8. Olivares M, Hertrampf E, Capurro MT, Wegner D. Prevalence of anemia in elderly subjects living at home: role of micronutrient deficiency and inflammation. Eur J Clin Nutr. 2000; 54: 834-9.

9. Chaves PHM, Xue Q, Guralnik JM, Ferrucci L, Volpato S, Fried LP. What constitutes normal hemoglobin concentration in community-dwelling disabled older women? J Am Geriatr Soc 2004; 52:1811-6 10 Allen LH. Causes of vitamin $B 12$ and folate deficiency. Food Nutr Bull. 2008; 29 (2): 20-34.

10. Antony AC. Megaloblastic anemias. In: Hoffman R, Benz EJ Jr, Silberstein LE, et al. Hematology: Basic Principles and Practice. Philadelphia. Elsevier. 2012.

11. Stabler SP, Allen RH, Savage DG, Linderbaum J. Clinical spectrum and diagnosis of cobalamin deficiency. Blood. 1990; 76 (5): 871-81.

12. Nygard O, Nordrehaug JE, Refsum H, Ueland PM, Farstad $M$, Vollset SE. Plasma homocysteine levels and mortality in patients with coronary artery disease. $N$ Engl J Med. 1997; 337: 230-6.

13. British Medical Association, Royal Pharmaceutical Society of Great Britain. British National Formulary. 2004.

14. Middleton J, Wells W. Vitamin B12 injections: considerable source of work for the district nurse. British Med J Clin Res Ed 1985.

15. Elia M. Oral or parenteral therapy for B12 deficiency. The 
Lancet 1998.

16. Lederle FA. Oral cobalamin for pernicious anemia. Medicine's best kept secret? J Am Med Assoc. 1991;265:94-5.

17. Antony AC. Megaloblastic anemias. In: Hoffman R, Benz EJ $J r$, Shattil SJ, Furie B, Cohen HJ, Silberstein LE, McGlave P, eds. Hematology: basic principles and practice. 3rd ed. New York, NY: Churchill Livingston; 2000. p 446-85.

18. Ross $G$, Mollin D, Cox E, Ungley C. Hematologic responses and concentration of vitamin B12 in serum and urine following oral administration of vitamin B12 without intrinsic factor. Blood 1954; 9: 473-88.

19. Kaferle J, Strzoda CE. Evaluation of macrocytosis. Am Fam Physi. 2009;79:203-8.

20. Cohen HJ. Anemia in the elderly: Clinical impact and diagnosis. J Am Geriatr Soc. 2003, 51:1-26.

21. Pennix BEJH, Pahor M, Cesari M, Corsi AM, Woodman RC, Bandinelli S, Guralnik JM, Ferrucci L. Anemia is associated with disability and decreased physical performance and muscle strength in the elderly. J Am Geriatr Soc. 2004; 52: 719-24.

22. Salive $M E$, Cornoni-Huntley J, Guralnik JM, Phillips $C L$, Wallace RB, Ostfeld AM, Cohen HJ. Anemia and hemoglobin levels in older persons: relationship with age, gender, and health status. J Am Geriatr Soc 1992; 40: 489-96.

23. Carmel R. Cobalamin, the stomach, and aging. Am J Clin Nutr. 1997; 66 (4): 750-9.

24. Donadelli SP, Junqueira-Franco MV, de Mattos Donadelli $C A$, Salgado W Jr, Ceneviva R, Marchini JS, et al. Daily vitamin supplementation and hipovitaminosis after obesity surgery. Nutrition 2011; 28 (4): 391-6.

25. Higgins JPT, Green S (editors). Cochrane Handbook for Systematic Reviews of Interventions Version 5.0.2 [updated September 2009]. The Cochrane Collaboration, 2009.

26. PEDro (Physiotherapy Evidence Database). (consultado 30/11/2012). Disponible en:http://www.pedro.fhs.usyd. edu.au/spanish/scale_item_spanish.htlm.

27. Maher CG. A systematic review of workplace interventions to prevent low back pain. Aust J Physiother. 2000;46:25969.

28. Maher CG, Sherrington $C$, Herbert RD, Moseley AM, Elkins $M$ : Reliability of the PEDro scale for rating quality of ran- domized controlled trials. Phys Ther. 2003;83(8):713-21.

29. Elkins $M R$, Herbert RD, Moseley AM, Sherrington $C$, Maher $C$. Rating the quality of trials in systematic reviews of physical therapy interventions. Cardiopulm Phys Ther J. 2010;21:20-6.

30. Kuzminski AM, Del Giacco EJ, Allen RH, et al. Effective treatment of cobalamin Blood. 1998;92:1191-8.

31. Boloman Z, Kadikoylu G, Yukselen V, et al. Oral versus intramuscular cobalamin treatment in megaloblastic anemia: a single-center, prospective, randomized open-labe study. Clin Ther.2003;25:3124-313.

32. Sanz-Cuesta et al. Oral versus intramuscular administration of vitamin $B 12$ for the treatment of patients with vitamin B12 deficiency: a pragmatic, randomised, multicentre, non-inferiority clinical trial undertaken in the primary healthcare setting BMC Public Health.2012:12;394.

33. Castelli M, Friedman K, Sherry J, Brazzillo K, Genoble $L$, Bhargava $P$, Riley MG. Comparing the efficacy and tolerability of a new daily oral vitamin $B 12$ formulation and intermittent intramuscular vitamin $B 12$ in normalizing low cobalamin levels: a randomized, open-label, parallel-group study. Clin Ther. 2011 Mar;33(3):358-71.

34. Van Tulder M, Furlan A, Bombardier C, Bouter L. Editorial board of the Cochrane Collaboration back review group. Updated method guidelines for systematic reviews in the Cochrane Collaboration back review group. Spine. 2003;28:1290-9.

35. Butler CC, Vidal-Alaball J, Cannings-John R, et al. Oral vitamin $B 12$ versus intramuscular vitamin $B 12$ for vitamin $B 12$ deficiency: a systematic review of randomized controlled trials. Fam Pract. 2006; 23: 279-85.

36. Eli M. Oral or parenteral therapy for B12 deficiency. Lancet 1998;352:1721-2.

37. Van Walraven C, Austin P, Nalylor CD. Vitamin B12 injections versus oral supplements. How much money could be saved by switching from injections to pills. Can Fam Physi 2001;47:79-86.

38. Kwong JC, Carr D, Dhalla IA, Tom-Kun D, Upshur REG. Oral Vitamin $B 12$ therapy in the primary care setting: $A$ qualitative and quantitative study of patients perspectives. BMC Fam Pract. 2005;6:8-17. 